\title{
Reactive infectious mucocutaneous eruptions associated with SARS-CoV-2 infection
}

\author{
Shaji Thomas John*, Gayathri K., Rizwana P., Abhay M. Martin
}

Department of Paediatrics and Dermatology, Baby Memorial Hospital, Calicut, Kerala, India

Received: 07 November 2021

Accepted: 30 November 2021

*Correspondence:

Dr. Shaji T. John,

E-mail: doctorshaji@hotmail.com

Copyright: (c) the author(s), publisher and licensee Medip Academy. This is an open-access article distributed under the terms of the Creative Commons Attribution Non-Commercial License, which permits unrestricted non-commercial use, distribution, and reproduction in any medium, provided the original work is properly cited.

\section{ABSTRACT}

Several dermatological manifestations are described in COVID-19. A 3-year-old boy presented with fever, painful oral ulcers with COVID-19 infection. A diagnosis of reactive infectious mucocutaneous eruptions (RIME) was made. He was worked up for the usual causes of RIME but were all negative. His severe acute respiratory syndrome corona virus2 immunoglobulin M (SARS-CoV-2 IgM) was positive and he was managed with Intravenous hydrocortisone with which he improved dramatically. RIME might be the first/only manifestation of COVID-19 and it should be kept in mind.

Keywords: COVID-19, Reactive infectious mucocutaneous eruptions

\section{INTRODUCTION}

The World Health Organization (WHO) declared coronavirus disease (COVID-19) caused by severe acute respiratory syndrome coronavirus-2 (SARS-CoV-2) as a pandemic in March 2020. Patients with SARS-CoV-2 infection may present with various manifestations like fever, flu like symptoms, cough, respiratory distress syndrome, gastrointestinal symptoms, cardiovascular complications and mucocutaneous lesions.

SARS-CoV-2 enters and invades host cells via the angiotensin converting enzyme-2 (ACE-2) membrane receptor. The single cell RNA-seq (scRNA-Seq) studies of ACE-2 expression have detected high levels of expression in keratinized epithelial cell surfaces of the oral cavity, such as the dorsum of the tongue and hard palate. ${ }^{1}$ Viral infections disrupt epithelial cells and trigger local inflammatory reactions that are abrupt in onset. In the oral mucosa they present as solitary or multiple blisters or ulcerations. ${ }^{2}$ Around seven clinical patterns have been identified as the dermatologic manifestations of COVID19: morbilliform, pernio-like, urticarial, macular, erythematous, vesicular, papulo-squamous, and retiform purpura. ${ }^{3}$ The most common sites of involvement are the tongue $(38 \%)$, labial mucosa $(26 \%)$, and palate $(22 \%){ }^{4}$

\section{CASE REPORT}

A previously healthy 3 -year-old male child presented with history of high-grade fever and pain in the oral cavity and odynophagia of 5 days duration. The eruptions started as a small elevated lesions on the lower lip which progressed over a few days into small vesicles. These ruptured into painful erosions which subsequently bled and formed haemorrhagic crusts and purulent ulcers. The eruptions were accompanied by fever and difficulty in swallowing. There was no preceding history of drug intake, consumption of food or artificial colours or infectious illness. There was also no history of cough, breathing difficulty, rhinorrhoea, or gastrointestinal symptoms at any time. There was also no family history of similar illness.

At the time of presentation, he had high grade fever (102.6 $\left.{ }^{0} \mathrm{~F}\right)$, but was not in distress. His vital signs were normal. Dermatologic examination revealed confluent hemorrhagic crusted erosions on the upper and lower lips, hard and soft palate, cheeks and tongue. The lesions were 
tender to touch and bled easily. There was purulent discharge at the base of some erosions and had salivary drool due to difficulty in swallowing. There was no regional lymphadenopathy. There were discrete solitary vesicles on the wrist, foot and lower back. Systemic examination was unremarkable.

Based on the above clinical presentation, clinical possibilities of fixed eruptions (drug related and non-drug related), herpes simplex infection associated erythema multiforme (HAEM) and reactive infectious mucocutaneous eruption (RIME) were considered. Laboratory tests showed normal leucocyte count, liver function test and C-reactive protein (CRP). Tzanck smear test was negative for multinucleated giant cells. IgM serology for Herpes simplex virus (HSV) type 1 (most likely agent for Herpes labialis in a child) and polymerase chain reaction (PCR) test for HSV type 1 and type 2 were also negative. Hence Herpes virus infection was ruled out.

SARS CoV-2 infection was considered as another etiologic possibility. COVID reverse transcriptasepolymerase chain reaction (RT-PCR), D-dimer, Serum ferritin levels and SARS CoV-2 antibody tests were done. SARS-CoV-2 IgM was detected to be positive which suggested a recent infection. Mycoplasma infection was yet another etiology considered, but IgM mycoplasma was negative.

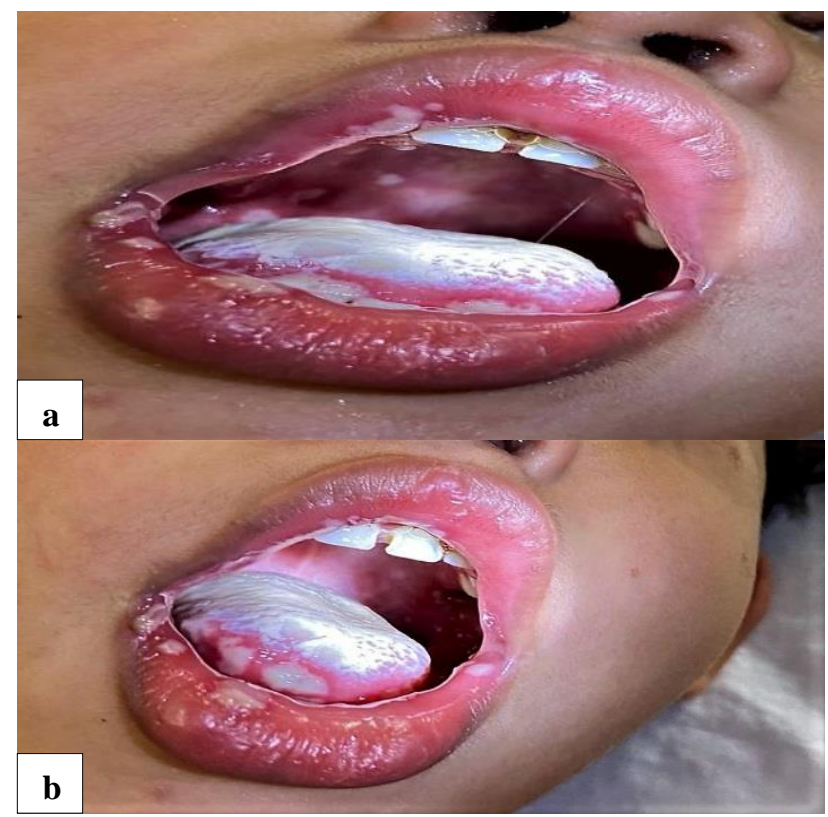

Figure 1: (a) Photo of the oral cavity showing lesions over lips, tongue and palate at the time of admission, and (b) photo of the oral cavity highlighting the lesions on the tongue at the time of admission.

With a working diagnosis of HAEM, systemic acyclovir was started initially. However, it was stopped when HSV reports turned negative. He was noted to have progressive worsening of oral pain and irritability. Hence, considering the possibility of SARS CoV-2, he was started on intravenous steroids (hydrocortisone) and he showed dramatic improvement in his mucositis.

In view of the clinical presentation and lab findings, a diagnosis of RIME due to SARS CoV-2 was made.

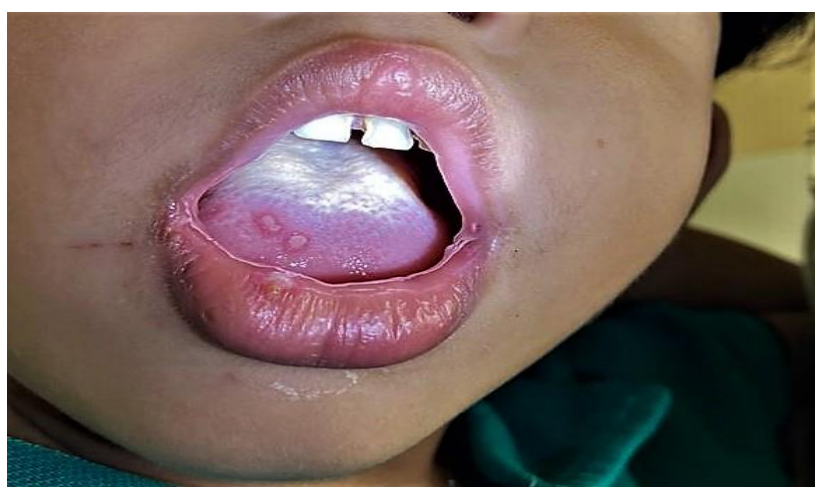

Figure 2: Photo of the lesion on the third day after the administration of hydrocortisone, showing resolution of the lesions on the lips and tongue.

\section{DISCUSSION}

RIME (previously known as mycoplasma-induced rash and mucositis) has arisen as the preferred terminology to include mucocutaneous eruptions that are caused by other infectious agents. ${ }^{5}$ It typically occurs as a late manifestation of exposure to mycoplasma and other infectious agents. RIME typically describes the clinical presentation of significant mucositis (oral, ocular, and anogenital) with only limited cutaneous involvement.

The association between RIME and SARS-CoV-2 has not been widely reported. On review of literature, only two cases of RIME have been reported in children. ${ }^{6}$ Both of them were in adolescents. They presented with fever, cough and had conjunctivitis and ulcerations over oral mucosa and glans penis. Their CRP was elevated with normal counts and they were SARS-COV-2 PCR positive at the time of admission. Both of them were treated with hydrocortisone, IVF, and analgesics. They had complete resolution of mucositis 1 week after discharge. Their clinical presentation was similar to our case. The combination of confirmed recent SARS-CoV-2 infection, absence of other concurrent clinical or laboratoryconfirmed infections, and prominent mucositis suggests SARS-CoV-2 as an infectious trigger for RIME.

\section{CONCLUSION}

Clinicians should be aware that SARS-CoV-2 may precipitate RIME and that systemic corticosteroids may be of benefit.

\section{ACKNOWLEDGEMENTS}

Authors would like to thank Dr. K. G. Alexander, Chairman and Managing Director, Baby Memorial 
Hospital, Calicut for permitting to submit this case for publication.

Funding: No funding sources

Conflict of interest: None declared

Ethical approval: Not required

\section{REFERENCES}

1. Xu H, Zhong L, Deng J, Peng J, Dan H, Zeng X, et al. High expression of ACE2 receptor of 2019-nCoV on the epithelial cells of oral mucosa. Int J Oral Sci. 2020;12(1):8.

2. Santosh ABR, Muddana K. Viral infections of oral cavity. J Family Med Prim Care. 2020;9:36-42.

3. Freeman EE, McMahon DE, Lipoff JB, Rosenbach M, Kovarik C, Desai SR, et al. The spectrum of COVID-19-associated dermatologic manifestations: An international registry of 716 patients from 31 countries. J Am Acad Dermatol. 2020;83(4):111829.
4. Amorim Dos Santos J, Normando AGC, Carvalho da Silva RL, De Paula RM, Cembranel AC, SantosSilva AR, et al. Oral mucosal lesions in a COVID-19 patient: New signs or secondary manifestations? Int J Infect Dis. 2020;97:326-8.

5. Ramien ML, Bruckner AL. Mucocutaneous eruptions in acutely ill pediatric patients - think of mycoplasma pneumoniae (and other infections) first. JAMA Dermatol. 2020;156(2):124-5.

6. Song A, Nicholson C, Maguiness S. Recurrent reactive infectious mucocutaneous eruption (RIME) in two adolescents triggered by several distinct pathogens including SARS-CoV-2 and influenza A. Pediatr Dermatol. 2021;00:1-4.

Cite this article as: John ST, Gayathri K, Rizwana P, Martin AM. Reactive infectious mucocutaneous eruptions associated with SARS-CoV-2 infection. Int J Contemp Pediatr 2022;9:132-4. 